\title{
On the geometry of entangled states.
}

\author{
Frank Verstraete, Jeroen Dehaene, Bart De Moor \\ Katholieke Universiteit Leuven, Department of Electrical Engineering, Research Group SISTA \\ Kard. Mercierlaan 94, B-3001 Leuven, Belgium
}

\begin{abstract}
The basic question that is addressed in this paper is finding the closest separable state for a given entangled state, measured with the Hilbert Schmidt distance. While this problem is in general very hard, we show that the following strongly related problem can be solved: find the Hilbert Schmidt distance of an entangled state to the set of all partially transposed states. We prove that this latter distance can be expressed as a function of the negative eigenvalues of the partial transpose of the entangled state, and show how it is related to the distance of a state to the set of positive partially transposed states (PPT-states). We illustrate this by calculating the closest biseparable state to the $\mathrm{W}$-state, and give a simple and very general proof for the fact that the set of W-type states is not of measure zero. Next we show that all surfaces with states whose partial transposes have constant minimal negative eigenvalue are similar to the boundary of PPT states. We illustrate this with some examples on bipartite qubit states, where contours of constant negativity are plotted on two-dimensional intersections of the complete state space.
\end{abstract}

03.65.Bz

In this paper we try to get some insight into the geometrical structure of entangled states. The main goal will be to characterize the distance of an entangled state to the set of separable states. Related questions were addressed in the papers of Zyczkowski et al. 11 3], Pittenger et al. [4] and Witte et al. [5] (see also Ozawa [6]), although here we attack the problem from a different perspective.

The concept of negativity will turn out to be very much related to the Hilbert-Schmidt distance of a state to the set of separable states. It originates from the observation due to Peres [7] that taking a partial transpose of a density matrix associated with a separable state is still a valid density matrix and thus positive (semi)definite. Subsequently M.Horodecki,P.Horodecki and R.Horodecki [8] proved that this was a necessary and sufficient condition for a state to be separable if the dimension of the Hilbert space does not exceed 6 . In higher dimensional systems, no easy way of determining the separability of a state exists due to the existence of bound entangled states. We will therefore content ourselves to calculate the Hilbert Shmidt distance of an entangled state to the set of PPT-states (Remark that in the case of two qubits no bound entangled states exist).

This problem is highly related to calculating the distance of an entangled state to the set of partially transposed states, as the intersection of the set of all states with the set of all partially transposed states is equal to the set of all PPT-states. This is visualized in figure (11), where the boundary of the convex set of states $H$ consists of rank deficicient states. The set of partially transposed states is completely isomorf with the set of states, and can be seen as some kind of reflection of the set of states. The intersection of both sets is the convex set of PPT-states.

From figure (11) it is immediatly clear that the distance of an entangled state to the PPT ones is equal to the distance of an entangled state to the set of partially transposed states iff the closest partially transposed state is positive (semi)-definite; this condition will turn out to be almost always true.

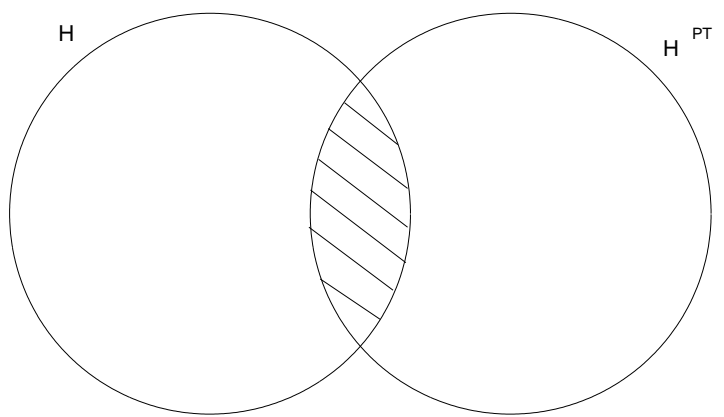

FIG. 1. The set of all states is depicted by $H$ and the set of all partial transposed states by $H^{P T}$. The intersection of both is the set of all PPT-states.

Let us now calculate the closest partially transposed state to an entangled state. The key observation is the fact that the Hilbert Schmidt norm is preserved under the partial transpose map. Therefore the proposed measure can be defined in the space of partial transposed density matrices as the minimal Hilbert Schmidt distance of $\rho^{P T}$ to the surface of positive (semi)-definite matrices with trace 1 , this surface being the partial transpose of the boundary of PPT-states.

We are therefore looking for the best positive semidefinite approximation of the indefinite matrix $\rho^{P T}$ in the Hilbert-Schmidt norm:

$$
\min _{\sigma \in H}\left\|\rho^{P T}-\sigma\right\|_{2}=\sqrt{\operatorname{Tr}\left(\left(\rho^{P T}-\sigma\right)^{2}\right)}
$$

Writing the eigenvalue decomposition $\rho^{P T}=U D U^{\dagger}$, and absorbing $U$ in $\sigma$, this problem is equivalent to finding 
$\sigma$ such that $\|D-\sigma\|$ is minimal. Using the eigenvalue decomposition $\sigma=V E^{2} V^{\dagger}$ with $\operatorname{Tr}\left(E^{2}\right)=1$, this can be written as a Lagrange constrained problem with cost function:

$$
K=\left\|D-V E^{2} V^{\dagger}\right\|_{2}-\lambda\left(\operatorname{Tr}\left(E^{2}\right)-1\right)
$$

It is immediatly clear that the optimal unitary $V$ is given by the identity: a positive definite matrix remains positive definite if off-diagonal elements are made zero. Differentiation leads to the result that the $e_{i}^{2}$ are either equal to 0 , either equal to $d_{i}+\lambda$. Normalization fixes the value of $\lambda$. Straightforward calculations show that th $e_{j}^{2}$ corresponding to the negative eigenvalues $d_{j}$ have to be choosen equal to zero and the other ones either equal to $d_{i}+\lambda$ either equal to 0 , depending on the sign of $d_{i}+\lambda$. The algorithm for finding the closest partially transposed state therefore becomes:

1. Calculate the eigenvalue decomposition of $\rho^{P T}=$ $U D U^{\dagger}$

2. Define $E^{2}$ as the unique diagonal positive (semi)definite normalized matrix such that its elements are $e_{i}^{2}=d_{i}+\lambda$ or $e_{i}^{2}=0$.

3. The closest partially transposed state $\rho_{s}$ is given by $\rho_{s}=\left(U E^{2} U^{\dagger}\right)^{P T}$. The Hilbert Schmidt distance between both states is given by

$$
\left\|\rho-\rho_{s}\right\|_{2}=\sqrt{\frac{\left(\sum_{i \in I_{p}} d_{i}+\sum_{i \in I_{n}} d_{i}\right)^{2}}{n_{p}}+\sum_{i \in I_{n}} d_{i}^{2}},
$$

where $I_{n}$ is the set of all indices corresponding to the negative eigenvalues of $\rho^{P T}, I_{p}$ is the set of indices corresponding to positive eigenvalues of $\rho^{P T}$ but for which $E_{i}^{2}=0$, and $n_{p}$ denotes the rank of $E^{2}$.

If $\rho_{s}$ is a state, it is guaranteed to be the closest PPTstate. Numerical investigations show that for example in the two qubit case the positiveness of $\rho_{s}$ happens in approximately $97 \%$ of the cases. If $\rho_{s}$ is not positive, then the distance to the set of partially transposed states calculated is a (fairly good) lower bound on the distance of the entangled state to the set of PPT-states.

Let us illustrate the above procedure with an example. Say we want to find the closest biseparable $2 \times 4$ state to the three qubit $W$-state [10] $|W\rangle=(|001\rangle+$ $|010\rangle+|100\rangle) / \sqrt{3}$. The eigenvalue decomposition of $(|W\rangle\langle W|)^{P T}=U D U^{\dagger}$, with the partial transpose operation taken over the 4-dimensional Hilbert space, is given by:

$D=\operatorname{diag}\left(\begin{array}{llllllll}2 / 3 & \sqrt{2} / 3 & 1 / 3 & 0 & 0 & 0 & 0 & -\sqrt{2} / 3\end{array}\right)$

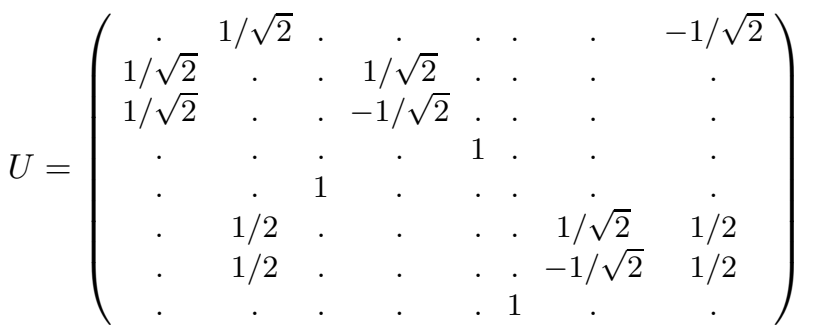

The eigenvalues $E^{2}$ are readily obtained:

$E^{2}=\operatorname{diag}\left(\begin{array}{llllllll}2 / 3-\sqrt{2} / 9 & 2 \sqrt{2} / 9 & 1 / 3-\sqrt{2} / 9 & 0 & 0 & 0 & 0 & 0\end{array}\right)$

Taking the partial transpose leads to the state $\rho_{s}$, where we used the notation $c=\sqrt{2} / 18$ :

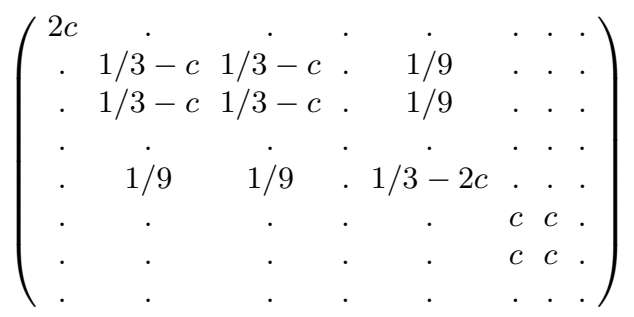

The eigenvalues of $\rho_{s}$ are non-negative and it is possible to show that $\rho_{s}$ is separable. We have therefore found the closest biseparable $2 \times 4$ state to the $|W\rangle$ state, and the Hilbert Schmidt distance to it is equal to $(2 / 3)^{3 / 2}$. Recently, the question arised whether the set of $\mathrm{W}$-type states is of measure zero. Using the language of the Hilbert-Schmidt distance, this problem is readily solved. Indeed, the question is solved if we can prove that the state obtained by mixing the W-state with a small random completely separable mixed state remains outside the set of all convex combinations of biseparable states (with relation to whatever partition). As there is no biseparable pure state infinitesimally close to the W-state, and a mixed state not infinitesimally close to a pure state is always at a finite distance from whatever pure state, it is proved that the set of W-type states is indeed not of measure zero. A different proof was given by Acin et al. [11]. Remark that the above proof is very general and can be used in systems of arbitrary dimensions: whenever there exists a pure state $\psi_{1}$ that can probabilistically be converted into another one $\psi_{2}$ but not vice-versa, the set of $\psi_{1}$-like states minus the set of the $\psi_{2}$-like states is of finite measure if there does not exist a $\psi_{2}$-like state infinitesimally close to $\psi_{1}$ !

The concept of negativity is also connected to the concept of robustness of entanglement 12]. Indeed, let us calculate how much an entangled bipartite state of whatever dimension has to be mixed with the identity before it gets PPT. In analogy with the previous derivation of the Hilbert-Schmidt distance, this amounts to the equivalent problem of how much one has to mix the partial transpose of $\rho$ with the identity before it gets positive semi-definite: 


$$
\min _{t}(1-t) \rho^{P T}+\frac{t}{4} I_{4} \geq 0
$$

This problem is readily solved, and the solution is

$$
t=\frac{\left|d_{\min }\right|}{\left|d_{\min }\right|+\frac{1}{4}}
$$

where $d_{\min }$ is the minimal negative eigenvalue of $\rho^{P T}$. The minimal $t$ is therefore only a function of the negative eigenvalues. A geometrical implication of this fact is that all surfaces of constant $d_{\min }$ are similar to the boundary of separable and entangled states: the set of all states with constant $d_{\text {min }}$ can be generated by extrapolating all lines from the identity to the boundary of separable states such that the distance of the extrapolated state to the identity is a constant factor $(>1)$ of the distance of the separable state to the identity.

Let us now move to the case of two qubits. In this case $\rho^{P T}$ has at most one negative eigenvalue [9]. Numerical investigations indicate that in a vast majority of the states the optimal rank of $E^{2}$ is equal to three, and if the rank is equal to two it implies that $\rho_{s}$ has a negative eigenvalue. For the states for which $E^{2}$ is rank 3, it follows that their distance to the set of partially transposed states is given by

$$
\left\|\rho-\rho_{s}\right\|=\frac{2}{\sqrt{3}}\left|d_{\min }\right|
$$

where $d_{\min }$ is the negative eigenvalue of $\rho^{P T}$. Surfaces of two-qubit states with constant negativity, defined as $N=2\left|d_{\text {min }}\right|$, have therefore two distinct properties: they are all similar to each other and the Hilbert-Schmidt distance between them is almost everywhere constant.

Let us illustrate the above findings by explicitely calculating some two-dimensional intersections of the set of all bipartite qubit states including the maximally mixed state. In the following figures we use the metric based on the Hilbert-Schmidt distance $\left\|\rho_{1}-\rho_{2}\right\|^{2}=$ $\operatorname{Tr}\left(\left(\rho_{1}-\rho_{2}\right)^{\dagger}\left(\rho_{1}-\rho_{2}\right)\right)$, and directions represented orthogonal to each other are orthogonal in the sense that $\operatorname{Tr}\left(A_{1} A_{2}\right)=0$. Rank deficient density operators always lie on the boundary of the intersection.

Note that an explicit parameterization of the boundary between the entangled and separable states can easily be obtained: it is at most a quartic function of the mixing parameters of the states, as their analytic expression can be obtained by setting the determinant of the partial transpose equal to zero.

As a first example we consider the plane containing the maximally mixed state and the states

$$
\begin{aligned}
& \rho_{1}=\frac{1}{2}\left(\begin{array}{l}
0 \\
1 \\
1 \\
0
\end{array}\right)\left(\begin{array}{llll}
0 & 1 & 1 & 0
\end{array}\right) \\
& \rho_{2}=\left(\begin{array}{l}
1 \\
0 \\
0 \\
0
\end{array}\right)\left(\begin{array}{llll}
1 & 0 & 0 & 0
\end{array}\right)
\end{aligned}
$$

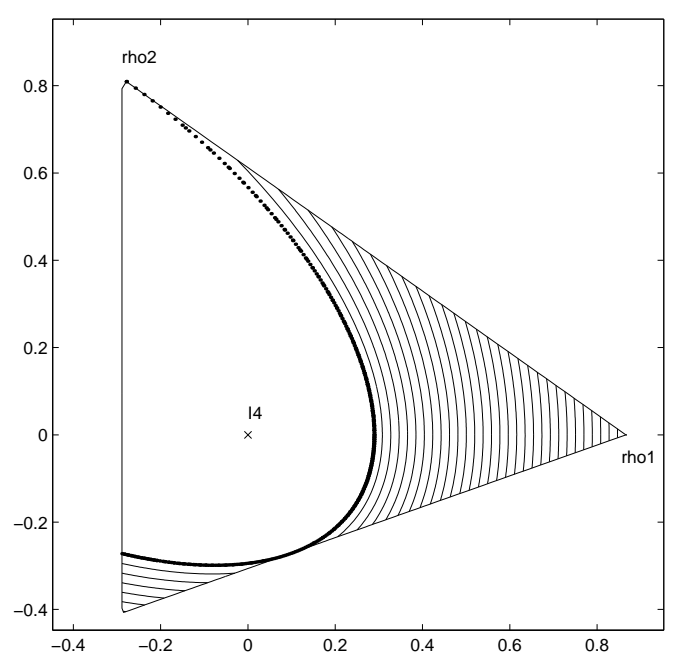

FIG. 2. Intersection of the convex set of all states including states (11) and the maximally mixed state. The contours represent surfaces of constant negativity, the starred line is the boundary between separable and entangled states.

The plane is plotted in figure (2) and the boundary of all (rank-deficient) states is given by the solid enveloppe. The starred line is the boundary between the convex set of separable states and the convex set of all states. The surfaces of constant negativity are indeed all similar to this boundary. The fact that the distance between these surfaces is not constant throughout the picture indicates that the closest separable states lie in other planes. Note that the Werner states lie along the line between the maximally mixed state and the maximally entangled state $\rho_{1}$. The thirth extremal point in the undermost left corner is given by the rank 2 state

$$
\rho=\left(\begin{array}{cccc}
\cdot & . & \cdot & \cdot \\
\cdot & \frac{1}{4} & -\frac{1}{4} & \cdot \\
\cdot & -\frac{1}{4} & \frac{1}{4} & \cdot \\
\cdot & \cdot & \cdot & \frac{1}{2}
\end{array}\right)
$$

This state is called a quasi-distillable state and has some remarkable properties: a single copy of it can be destilled infinitesimally close to the singlet state [13,14, it is the state with minimal negativity for given entanglement of formation [9], and it has furthermore the strange property that no global unitary operation can increase its entanglement 15.

Let us now consider a different plane including the maximally mixed state and

$$
\begin{aligned}
& \rho_{1}=\frac{1}{2}\left(\begin{array}{l}
0 \\
1 \\
1 \\
0
\end{array}\right)\left(\begin{array}{llll}
0 & 1 & 1 & 0
\end{array}\right) \\
& \rho_{2}=\frac{1}{2}\left(\begin{array}{l}
1 \\
1 \\
0 \\
0
\end{array}\right)\left(\begin{array}{llll}
1 & 1 & 0 & 0
\end{array}\right)
\end{aligned}
$$


This plane is obtained by rotating the previous plane around the axis $\rho_{1}-I_{4}$. In this case $\left(\rho_{1}-I_{4}\right)$ is orthogonal to $\left(\rho_{2}-I_{4}\right)$, and a completely different picture is obtained as shown in figure (3).

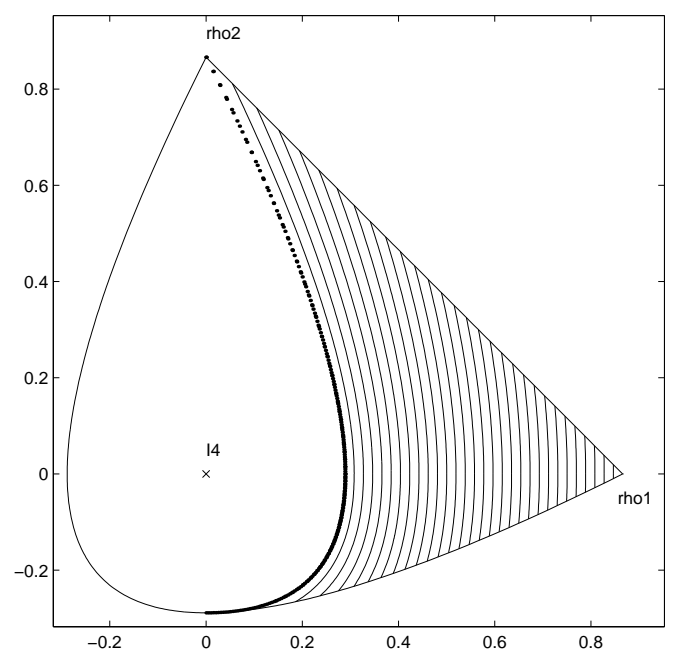

FIG. 3. Intersection of the convex set of all states including states (13) and the maximally mixed state.

Further rotation of the plane leads to the following states:

$$
\begin{aligned}
& \rho_{1}=\frac{1}{2}\left(\begin{array}{l}
0 \\
1 \\
1 \\
0
\end{array}\right)\left(\begin{array}{llll}
0 & 1 & 1 & 0
\end{array}\right) \\
& \rho_{2}=\frac{1}{2}\left(\begin{array}{l}
0 \\
1 \\
0 \\
0
\end{array}\right)\left(\begin{array}{llll}
0 & 1 & 0 & 0
\end{array}\right)
\end{aligned}
$$

The intersection of the state space by this plane is shown in figure (4).

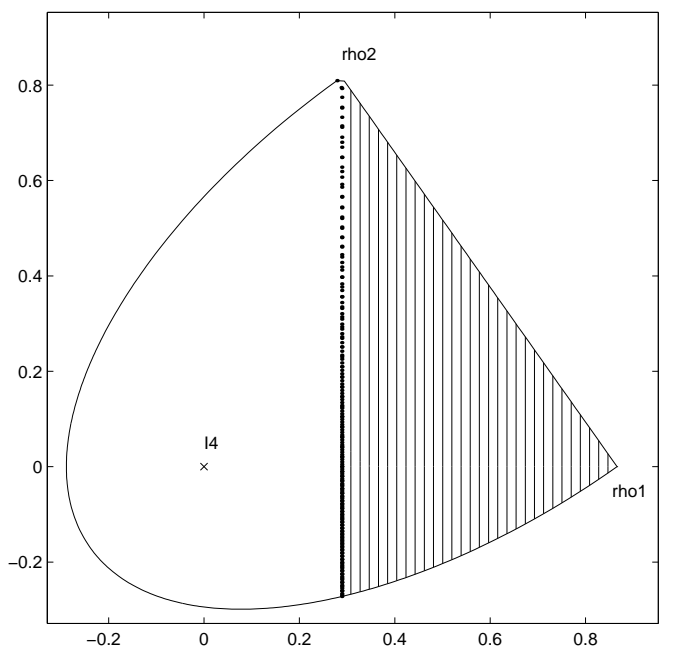

FIG. 4. Intersection of the convex set of all states including states (14) and the maximally mixed state.
The surfaces of constant negativity become straight lines, implying that the closest separable states lie in the same plane: the Hilbert-Schmidt distance betweem the surfaces of constant negativity has to be constant if they consist of parallel planes. Using the procedure previously outlined, it is indeed trivial to check that the separable state closest to the maximally entangled state $\rho_{1}$ lies in the defined plane and is given by

$$
\rho_{s}=\left(\begin{array}{cccc}
\frac{1}{6} & . & . & \cdot \\
\cdot & \frac{1}{3} & \frac{1}{6} & \cdot \\
\cdot & \frac{1}{6} & \frac{1}{3} & . \\
\cdot & \cdot & \cdot & \frac{1}{6}
\end{array}\right)
$$

Let us rotate the plane further over the $\left(\rho_{1}-I_{4}\right)$-axis:

$$
\begin{aligned}
& \rho_{1}=\frac{1}{2}\left(\begin{array}{l}
0 \\
1 \\
1 \\
0
\end{array}\right)\left(\begin{array}{llll}
0 & 1 & 1 & 0
\end{array}\right) \\
& \rho_{2}=\frac{1}{101}\left(\begin{array}{c}
10 \\
0 \\
0 \\
1
\end{array}\right)\left(\begin{array}{llll}
1 & 0 & 0 & 10
\end{array}\right)
\end{aligned}
$$

The resulting figure (5) combines the features of the previous figures. Three entangled disconnected regions arise, and once more we observe the strange shape of the boundary between entangled and separable states.

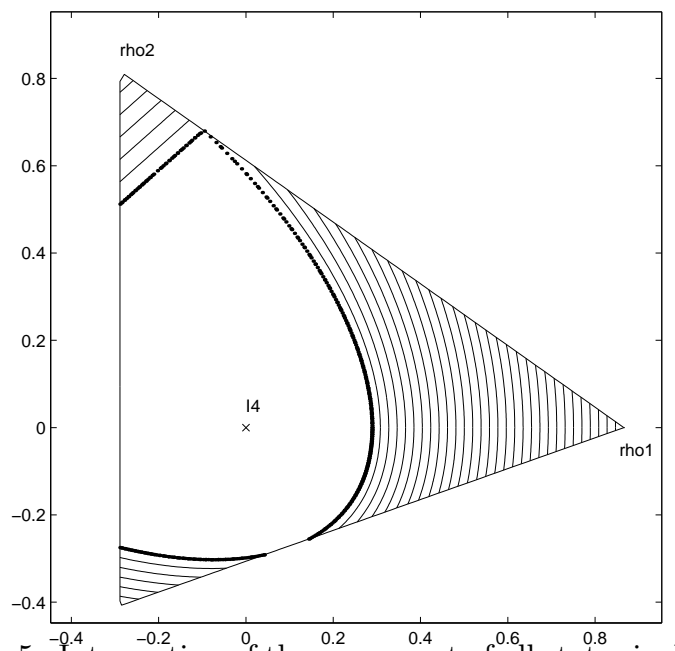

FIG. 5. Pntersection of the convex set of all states including states (16) and the maximally mixed state.

A plane with a highly symmetric contour lines is obtained if $\rho_{1}$ and $\rho_{2}$ are both taken to be maximally entangled states: 


$$
\begin{aligned}
& \rho_{1}=\frac{1}{2}\left(\begin{array}{l}
0 \\
1 \\
1 \\
0
\end{array}\right)\left(\begin{array}{llll}
0 & 1 & 1 & 0
\end{array}\right) \\
& \rho_{2}=\frac{1}{2}\left(\begin{array}{c}
0 \\
1 \\
-1 \\
0
\end{array}\right)\left(\begin{array}{llll}
0 & 1 & -1 & 0
\end{array}\right)
\end{aligned}
$$

Indeed, only straight lines are obtained in figure (6). The thirth extremal state is in this case given by $\rho=$ $\operatorname{diag}[1 / 2 ; 0 ; 0 ; 1 / 2]$.

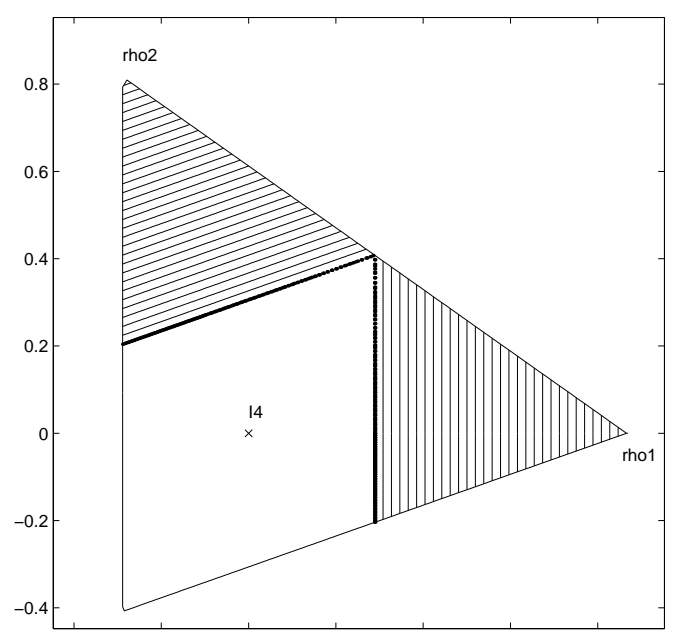

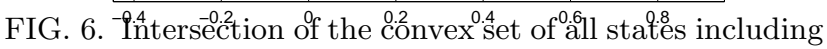
states $(17)$ and the maximally mixed state.

At last, we choose two random planes through the maximally mixed state and plot them in figure (7).

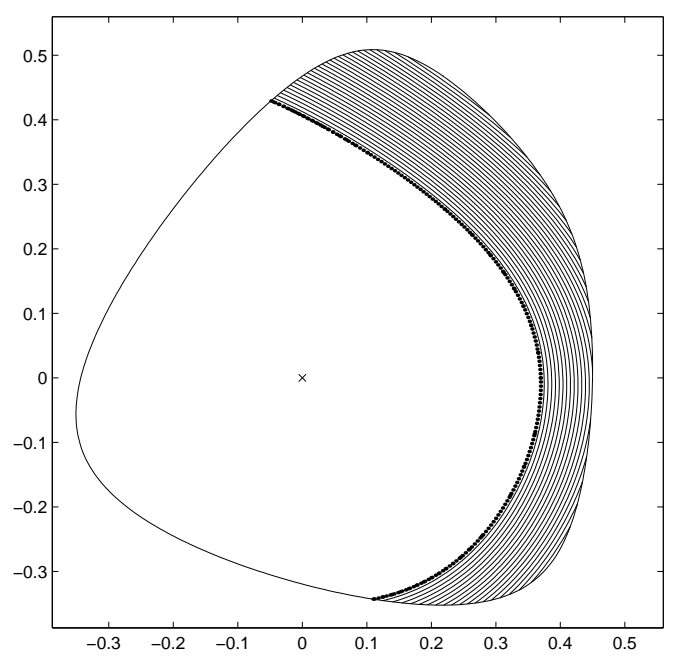

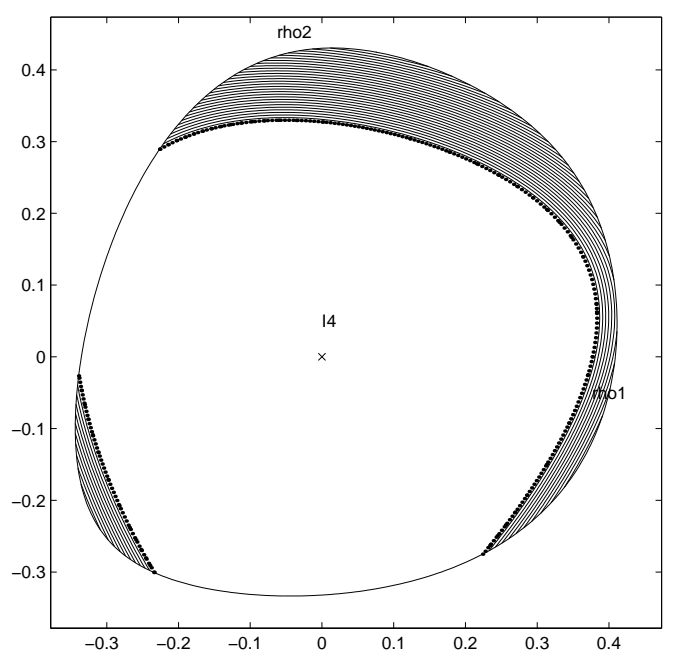

FIG. 7. Contour plots of the negativity on random planes including the maximally mixed state.

The similarity of all planes with constant negativity is clearly illustrated.

We acknowledge interesting discussions with $\mathrm{K}$. Audenaert, A. Pittenger and K. Zyczkowski, who gave the idea of considering 2-D intersections of the state space.

* frank.verstraete@esat.kuleuven.ac.be

\# jeroen.dehaene@esat.kuleuven.ac.be

\$ bart.demoor@esat.kuleuven.ac.be

[1] K. Zyczkowski, P. Horodecki, A. Sanpera, M. Lewenstein, Phys.Rev. A58, 883 (1998).

[2] K. Zyczkowski, W. Slomczynski, J.Phys. A31, 9095 (1998).

[3] K. Zyczkowski, Phys.Rev. A60, 3496 (1999).

[4] A. Pittenger and M. Rubin, quant-ph/0103038.

[5] C. Witte and M. Trucks, Phys.Lett. A257, 14 (1999).

[6] M. Ozawa, Phys. Lett. A268, 158 (2000).

[7] A. Peres, Physical Review Letters 76, 1413 (1996).

[8] M. Horodecki, P. Horodecki and R. Horodecki, Phys. Lett. A 223, 1 (1996).

[9] F. Verstraete, K. Audenaert, J. Dehaene and B. De Moor, internal report.

[10] W. Dür, G. Vidal and J. I. Cirac, Phys. Rev. A 62, 062314 (2000).

[11] A. Acn, D. Bru, M. Lewenstein, and A. Sanpera, Phys. Rev. Let. 87, 040401 (2001).

[12] G. Vidal and R. Tarrach, Phys.Rev. A59,141 (1999).

[13] M. Horodecki, P. Horodecki and R. Horodecki, Phys. Rev. A 60, 1888 (1999).

[14] F. Verstraete, J. Dehaene and Bart De Moor, Physical Review A 64, 010101(R) (2001).

[15] F. Verstraete, K. Audenaert and Bart De Moor, Physical Review A 64, 012316 (2001). 